\section{PREVALÊNCIA DA INADEQUAÇÃO NO CONSUMO DE NUTRIENTES ENTRE GESTANTES ATENDIDAS EM UNIDADES BÁSICAS DE SAÚDE}

\author{
Prevalence of inadequacy in nutrient consumption among \\ pregnant women attended in basic health units \\ Prevalencia de la inadecuación del consumo de nutrientes por
embarazadas asistidas en unidades básicas de salud
}

\section{RESUMO}

Objetivo: Avaliar a prevalência da inadequação no consumo de nutrientes entre gestantes atendidas em Unidades Básicas de Saúde de Picos-PI, Brasil. Métodos: Estudo transversal envolvendo 72 gestantes. Coletaram-se dados sociodemográficos e de estilo de vida, além de informações sobre ingestão dietética, a partir de 3 recordatórios alimentares de 24 horas. Calcularam-se prevalências de inadequação para carboidrato, cálcio, ferro, vitaminas A, C, E e ácido fólico, baseando-se em suas respectivas médias estimadas (EAR); avaliouse a adequação da ingestão energética habitual em relação ao gasto energético usual indiretamente pelo índice de massa corporal (IMC). Resultados: Verificou-se que $86,1 \%$ $(n=62)$ das gestantes apresentavam renda per capita inferior a 1 salário mínimo, 4,2\% $(n=3)$ fumavam e 11,1\% ( $n=8)$ faziam uso de bebida alcoólica durante a gestação. No que se refere ao consumo, os carboidratos e a vitamina $\mathrm{C}$ tiveram prevalência de inadequação menor que $2 \%$, enquanto a vitamina A ficou entre $30 \%$ e 50\%. Cálcio, ferro, ácido fólico e vitamina $\mathrm{E}$ tiveram prevalência de inadequação maior que $98 \%$. Ao considerar que 38,9\% (n=28) das gestantes estavam com excesso de peso e $4,2 \%(n=3)$, com baixo peso, presume-se elevada prevalência de consumo inadequado de energia. Conclusão: Houve elevada prevalência de inadequação no consumo dos micronutrientes cálcio, ferro, ácido fólico e vitamina $\mathrm{E}$ nas gestantes, além de inadequação de consumo energético - situação preocupante, visto que pode desencadear consequências adversas para o binômio mãe-filho.

Descritores: Gestantes; Consumo Alimentar; Nutrientes; Estado Nutricional; Necessidades Nutricionais.

\section{ABSTRACT}

Objective: To assess the dietary intake of pregnant women attended in Basic Health Units of Picos-Piaui. Methods: Cross-sectional study involving 72 pregnant women. Sociodemographic and lifestyle data was collected, plus information on dietary intake from three 24-hour food recalls. The prevalence of inadequacy for carbohydrate, calcium, iron, $A, C, E$ vitamins, and folate were calculated based on their respective estimated average requirements (EAR), while the adequacy of habitual energy intake relative to usual energy expenditure was estimated indirectly by body mass index (BMI). Results: It was found that $86.1 \%$ of the pregnant women had a per capita income lower than one minimum wage, $4.2 \%$ were smokers and $11.1 \%$ used alcohol during pregnancy. As regards consumption, carbohydrates and vitamin $C$ had inadequacy prevalence lower than $2 \%$, while vitamin $A$ ranged from 30 to $50 \%$. Calcium, iron, folate, and vitamin $E$ had inadequacy prevalence higher than $98 \%$. Considering that $38.9 \%$ of the pregnant women were overweight and $4.2 \%$, underweight, it is assumed that the prevalence of energy intake inadequacy is high. Conclusion: There was a high inadequacy prevalence in the micronutrients calcium, iron, folate, and vitamin E intake in pregnant women, as well as high inadequacy of energy consumption - a disturbing situation, since it can trigger adverse consequences for the mother-baby binomial.

Descriptors: Pregnant Women; Food Consumption; Nutrients; Nutritional Status; Nutritional Requirements.
Artigo Original
1) Universidade Federal do Piauí - UFPI Picos (PI) - Brasil

2) Faculdade de Saúde Pública da Universidade de São Paulo - FSP - São Paulo (SP) - Brasil
Recebido em: 03/01/2014 Revisado em: 21/05/2014 Aceito em: 20/06/2014 


\section{RESUMEN}

Objetivo: Evaluar la prevalencia de la inadecuación del consumo de nutrientes por embarazadas asistidas en Unidades Básicas de Salud de Picos-PI, Brasil. Métodos: Estudio transversal con 72 embarazadas. Se recogieron datos sociodemográficos, del estilo de vida e informaciones sobre ingesta dietética a partir de 3 recordatorios de 24 horas. Se calculó las prevalencias de inadecuación para carbohidrato, calcio, hierro, vitaminas $A, C$, E y ácido fólico basándose en sus respectivas medias estimadas (EAR); se evaluó la adecuación de ingesta energética habitual respecto al gasto energético usual de modo indirecto a través del indice de masa corporal (IMC). Resultados: Se verificó que el $86,1 \%(n=62)$ de las embarazadas presentaban renta per capita menor que 1 sueldo mínimo, el 4,2\% (n=3) fumaban y el 11,1\% $(n=8)$ tomaban bebida alcohólica en el embarazo. Respecto al consumo, los carbohidratos y la vitamina $C$ tuvieron prevalencia de inadecuación menor que el 2\% mientras que la vitamina A se quedó entre el $30 \%$ y el $50 \%$. Calcio, hierro, ácido fólico y vitamina E tuvieron prevalencia de inadecuación mayor que el $98 \%$. Considerando que el 38,9\% $(n=28)$ de las embarazadas tenían exceso de peso y el 4,2\% (n=3) bajo peso, presúmase de elevada prevalencia del consumo inadecuado de energía. Conclusión: Hubo elevada prevalencia de inadecuación del consumo de los micronutrientes calcio, hierro, ácido fólico y vitamina $E$ en las embarazadas además de la inadecuación del consumo energéticosituación preocupante ya que puede desencadenar consecuencias adversas para el binomio madre-hijo.

Descriptores: Mujeres Embarazadas; Consumo de Alimentos; Nutrimentos; Estado Nutricional; Necessidades Nutricionales.

\section{INTRODUÇÃO}

Evidências sugerem que comportamentos saudáveis durante a gravidez envolvendo ingestão dietética adequada, prática de atividade física, ganho adequado de peso e não abuso de substâncias nocivas, tais como álcool e tabaco, podem contribuir para a boa saúde da mãe e do bebê $\hat{e}^{(1,2)}$. Em contrapartida, a nutrição materna inadequada tem sido associada ao nascimento de bebês pré-termos e com restrição de crescimento intrauterino ${ }^{(1-3)}$.

Assim, o estado nutricional materno, antes e durante a gestação, é um forte determinante para o desfecho da gestação e a saúde da mãe e do recém-nascido ${ }^{(1,3)}$. Entendese que o aporte energético inadequado durante a gravidez pode comprometer o crescimento fetal, assim como a deficiência de micronutrientes pode levar a consequências adversas para a gestante e para o desenvolvimento fetal ${ }^{(3,4)}$.

Nesse contexto, é primordial avaliar o consumo alimentar em gestantes, mesmo que essa avaliação não seja um indicador direto do estado nutricional, pois ela auxilia no diagnóstico nutricional, tornando possível perceber um risco subclínico para desfechos desfavoráveis da gestação, tais como prematuridade, baixo peso ao nascer, diabetes mellitus gestacional, pré-eclâmpsia, dentre outros, constituindo-se, assim, o primeiro indicador de risco nutricional ${ }^{(5,6)}$.

Apesar da relevância do consumo alimentar, não existe estudo para identificar situações alimentares e nutricionais de gestantes no estado do Piauí. Na tentativa de preencher essa lacuna, o presente estudo teve como objetivo avaliar a prevalência da inadequação no consumo de nutrientes entre gestantes atendidas em Unidades Básicas de Saúde de Picos-PI.

\section{MÉTODOS}

Tratou-se de estudo observacional, do tipo transversal, realizado com gestantes atendidas em Unidades Básicas de Saúde (USB) do município de Picos-PI, no período de março a maio de 2011.

Participaram gestantes de cinco Unidades Básicas de Saúde com maior fluxo de consultas pré-natal - em média de 19 gestantes adultas em atendimento por mês em cada UBS. Do total de 95 gestantes atendidas nesse período, foram incluídas 76 ( $80 \%$ da população de estudo), que se encontravam na sala de espera para consulta ou exame de pré-natal. Consideraram-se elegíveis aquelas de idade superior a vinte anos e em qualquer período da gestação. Excluíram-se as gestantes consideradas em risco (hipertensas, diabéticas, gemelares etc.) e com problemas mentais.

Das 76 elegíveis para o estudo, apenas $72(94,7 \%)$ concluíram todas as etapas. Uma desistiu do estudo, duas não responderam a um dos recordatórios de 24 horas e uma não respondeu adequadamente ao questionário socioeconômico.

Após leitura e esclarecimento do Termo de Consentimento Livre e Esclarecido, a equipe previamente treinada de acadêmicos em Nutrição aplicou os instrumentos.

Primeiramente, coletaram-se informações sociodemográficas (situação conjugal, ocupação materna, escolaridade materna, idade materna, idade gestacional e renda per capita) e de estilo de vida (hábito de fumar e uso de bebida alcoólica). Em seguida, obtiveram-se informações referentes ao consumo alimentar das gestantes, através da aplicação de três recordatórios alimentares de 24 horas (R24h) em dias alternados, compreendendo um dia no final de semana. Para tanto, utilizou-se formulário próprio, auxílio de álbum fotográfico de porções de alimentos e utensílios de cozinha, a fim de se coletar informações referentes aos horários das refeições, alimentos, preparações e porções. Não foram incluídos no estudo dados referentes ao uso de suplementos alimentares. 
Avaliou-se o consumo dos nutrientes com auxílio do software Nutwin 2002, versão 1.2, da Universidade Federal de São $\mathrm{Paulo}^{(7)}$, sendo inseridos os alimentos que não constavam no programa, a partir da Tabela Brasileira de Composição de Alimentos ${ }^{(8)}$.

Para as análises das prevalências de inadequação dos macronutrientes e micronutrientes, foram considerados os valores das Dietary Reference Intakes (DRI), propostos pelo Food and Nutrition Board (FNB) ${ }^{(9)}$, sendo a amostra reunida em um único grupo, pois os valores de referência recomendados para gestantes de 19 a 30 anos e 31 a 50 anos são os mesmos para todos os macronutrientes e micronutrientes analisados. Calcularam-se as prevalências de inadequação de carboidrato, cálcio, ferro, vitamina $\mathrm{A}$, vitamina $\mathrm{C}$, vitamina $\mathrm{E}$ e ácido fólico, baseando-se em suas respectivas necessidades médias estimadas $(\mathrm{EAR})^{(9,10)}$.

Para a estimativa da prevalência de inadequação da ingestão habitual, procedeu-se anteriormente ao ajuste pela variabilidade intrapessoal proposto pelo Institute Of Medicine $(\mathrm{IOM})^{(9)}$, utilizando-se análise de variância ANOVA com um fator de classificação ${ }^{(11)}$.

Para avaliar a adequação da ingestão energética, utilizou-se o Índice de Massa Corporal (IMC), considerado pelo IOM como o melhor indicador para essa análise. Para tanto, os valores de IMC dentro dos limites de eutrofia de 18,5 a $24,9 \mathrm{~kg} / \mathrm{m}^{2}$ (para adultos) indicaram ingestão energética adequada em relação ao gasto. Valores de IMC abaixo dos intervalos normais indicaram ingestão energética inadequada; acima dos intervalos normais, foram indicativos do excesso de ingestão energética.

Classificou-se o estado pré-gestacional de acordo com o IMC de mulher adulta, sendo considerado baixo peso IMC < $18,5 \mathrm{~kg} / \mathrm{m}^{2}$, eutrófica IMC entre 18,5 e $24,9 \mathrm{~kg} / \mathrm{m}^{2}$ e excesso de peso $\mathrm{IMC} \geq 25,0 \mathrm{~kg} / \mathrm{m}^{2}$; o gestacional foi classificado pelo IMC, segundo a $\mathrm{IOM}^{(12)}$, sendo considerado baixo peso IMC $<19,8 \mathrm{~kg} / \mathrm{m}^{2}$, eutrófica com IMC entre 19,8 e $26,0 \mathrm{~kg} / \mathrm{m}^{2}$ e excesso de peso IMC $>26,0 \mathrm{~kg} / \mathrm{m}^{2}$. A partir das informações do cartão da gestante, foram obtidos o peso pré-gestacional e a altura. $\mathrm{O}$ peso atual/gestacional foi aferido no momento da consulta, com a gestante usando roupas leves, em uma balança eletrônica com capacidade de 0 a $150 \mathrm{~kg}$ e precisão de $100 \mathrm{~g}$.

Realizou-se análise estatística com o auxílio do software SPSS, versão 17.0, sendo os valores expressos em frequência absoluta e relativa.

Tabela I - Descrição das gestantes atendidas em Unidades Básicas de Saúde de acordo com características sociodemográficas e estilo de vida. Picos-PI, 2011.

\begin{tabular}{|c|c|c|c|}
\hline Características & & $\mathbf{n}$ & $\%$ \\
\hline Situação conjugal & $\begin{array}{l}\text { Casada/mora com companheiro } \\
\text { Solteira/separada }\end{array}$ & $\begin{array}{l}60 \\
12\end{array}$ & $\begin{array}{l}83,3 \\
16,7\end{array}$ \\
\hline Ocupação & $\begin{array}{l}\text { Sim } \\
\text { Não }\end{array}$ & $\begin{array}{l}27 \\
45\end{array}$ & $\begin{array}{l}37,5 \\
62,5\end{array}$ \\
\hline Escolaridade & $\begin{array}{l}\text { Sem escolaridade } \\
\text { Ensino fundamental } \\
\text { Ensino médio } \\
\text { Ensino superior }\end{array}$ & $\begin{array}{c}3 \\
15 \\
43 \\
11\end{array}$ & $\begin{array}{c}4,2 \\
20,9 \\
59,7 \\
15,3\end{array}$ \\
\hline Faixa etária & $\begin{array}{l}20-34 \text { anos } \\
>35 \text { anos }\end{array}$ & $\begin{array}{c}68 \\
4\end{array}$ & $\begin{array}{c}94,4 \\
5,6\end{array}$ \\
\hline Idade gestacional & $\begin{array}{l}1^{\circ} \text { trimestre } \\
2^{\circ} \text { trimestre } \\
3^{\circ} \text { trimestre }\end{array}$ & $\begin{array}{c}9 \\
30 \\
33\end{array}$ & $\begin{array}{l}12,5 \\
41,7 \\
45,8\end{array}$ \\
\hline Renda per capita & $\begin{array}{l}\text { Até } \mathrm{R} \$ 200,00 \\
\mathrm{R} \$ 200,00 \text { a } \mathrm{R} \$ 545,00 \\
\text { Acima de } \mathrm{R} \$ 545,00^{*}\end{array}$ & $\begin{array}{l}32 \\
30 \\
10\end{array}$ & $\begin{array}{l}44,4 \\
41,7 \\
13,9\end{array}$ \\
\hline Fuma na gestação & $\begin{array}{l}\text { Sim } \\
\text { Não }\end{array}$ & $\begin{array}{c}3 \\
69\end{array}$ & $\begin{array}{c}4,2 \\
95,8\end{array}$ \\
\hline Uso de álcool na gestação & $\begin{array}{l}\text { Sim } \\
\text { Não }\end{array}$ & $\begin{array}{c}8 \\
64\end{array}$ & $\begin{array}{l}11,1 \\
88,9\end{array}$ \\
\hline Total & & 72 & 100,0 \\
\hline
\end{tabular}

*Salário mínimo R $\$ 545,00$ 
Este estudo foi aprovado pelo Comitê de Ética em Pesquisa da Universidade Federal do Piauí, de acordo com a Resolução 466/12 ${ }^{(13)}$, sob o protocolo $n^{\circ}$ 0462.0.045.00010.

\section{RESULTADOS}

O perfil das gestantes assistidas em Unidades Básicas de Saúde de Picos-PI mostrou que 68 (94,4\%) encontravamse na faixa etária entre 20 e 34 anos, e 33 (45,8\%) estavam no $3^{\circ}$ trimestre de gestação (Tabela I).

Com relação às características sociodemográficas e de estilo de vida, verificou-se maior prevalência de união estável $(\mathrm{n}=60 ; 83,3 \%)$, sem trabalho fora de casa $(\mathrm{n}=45$; $62,5 \%$ ) e renda per capita menor que um salário mínimo $(\mathrm{n}=62 ; 86,1 \%)$. Quanto à escolaridade, observou-se maior percentual de gestantes com ensino médio $(n=43 ; 59,7 \%)$. Além disso, constatou-se que $3(4,2 \%)$ gestantes fumavam e $8(11,1 \%)$ faziam uso de bebida alcoólica durante a gestação (Tabela I).

A partir dos valores de ingestão de energia e nutrientes ajustados pela variabilidade intrapessoal, apresentados na tabela II, foram estimadas as prevalências de inadequação na população estudada (Tabela III).

Foi possível observar que apenas o consumo de carboidratos e vitamina $\mathrm{C}$ mostrou inadequação menor que $2 \%$. A prevalência da inadequação no consumo de vitamina A foi de 30 a $50 \%$, enquanto para cálcio, ferro, vitamina E e ácido fólico, foi maior que 98\% (Tabela III).

A Figura 1 mostra que aproximadamente $40 \%$ das gestantes pesquisadas encontravam-se acima do peso desejável para a idade gestacional. Presume-se que o grupo estudado apresentou prevalência elevada do consumo inadequado de energia, com $4,2 \%(n=3)$ de baixo consumo e $38,9 \%(n=28)$ de consumo excessivo, segundo o estado nutricional na fase gestacional.

Tabela II - Descrição do consumo de macro e micronutrientes antes e após o ajuste pela variabilidade intrapessoal e respectivas razões de variâncias do consumo alimentar de gestantes atendidas em Unidades Básicas de Saúde. Picos-PI, 2011.

\begin{tabular}{lccccccccc}
\hline & \multicolumn{4}{c}{ Valores não ajustados } & \multicolumn{5}{c}{ Valores ajustados } \\
\cline { 2 - 10 } Nutrientes & Mín & Máx & Média & DP & Min & Máx & Média & DP & $\begin{array}{c}\text { Sb/ } \\
\text { Sobs }\end{array}$ \\
\hline Energia (kcal) & 900,6 & 2699,4 & 1600,3 & 386,1 & & & & & \\
Carboidratos $(\mathrm{g})$ & 136,8 & 448,2 & 238,3 & 61,3 & 152,5 & 415,6 & 238,3 & 51,8 & 0,845 \\
Proteínas $(\mathrm{g})$ & 35,1 & 135,4 & 72,8 & 19,1 & 42,52 & 123,1 & 72,80 & 15,37 & 0,804 \\
Lipídeos $(\mathrm{g})$ & 16,8 & 80,4 & 40,4 & 14,7 & 21,5 & 72,5 & 40,4 & 11,8 & 0,801 \\
Cálcio $(\mathrm{mg})$ & 151,9 & 885,1 & 419,5 & 175,2 & 192,3 & 814,8 & 419,5 & 148,7 & 0,849 \\
Ferro $(\mathrm{mg})$ & 5,9 & 19,7 & 12,8 & 3,2 & 7,7 & 17,9 & 12,8 & 2,4 & 0,737 \\
Vitamina A $(\mu \mathrm{g})$ & 76,8 & 4324,2 & 622,2 & 827,2 & 399,7 & 2132,6 & 622,2 & 337,5 & 0,408 \\
Vitamina E $(\mathrm{mg})$ & 0,9 & 7,8 & 3,3 & 1,5 & 1,5 & 6,7 & 3,3 & 1,1 & 0,761 \\
Vitamina C $(\mathrm{mg})$ & 0,9 & 4121,1 & 502,4 & 781,8 & 375,6 & 1418,0 & 502,4 & 197,8 & 0,253 \\
Ácido fólico $(\mu \mathrm{g})$ & 72,9 & 369,3 & 194,3 & 63,8 & 119,0 & 302,8 & 194,3 & 39,5 & 0,620 \\
\hline
\end{tabular}

*Sb/Sobs: razão de variância

Tabela III - Descrição das gestantes atendidas em Unidades Básicas de Saúde de acordo com as prevalências de inadequação no consumo de macro e micronutrientes. Picos-PI, 2011.

\begin{tabular}{lccc}
\hline Nutrientes & $\begin{array}{c}\text { EAR } \\
\text { (gestação 19-50 anos) }\end{array}$ & Valor $\mathbf{z}$ & $\begin{array}{c}\text { Prevalência de } \\
\text { inadequação }\end{array}$ \\
\hline Carboidratos $(\mathrm{g})$ & 135 & 2,0 & $<2 \%$ \\
Cálcio $(\mathrm{mg})$ & 800 & $-2,56$ & $>98 \%$ \\
Ferro $(\mathrm{mg})$ & 22 & $-3,85$ & $>98 \%$ \\
Vitamina A $(\mu \mathrm{g})$ & 550 & 0,21 & 30 a $50 \%$ \\
Vitamina E $(\mathrm{mg})$ & 12 & $-7,82$ & $>98 \%$ \\
Vitamina C $(\mathrm{mg})$ & 70 & 2,19 & $<2 \%$ \\
Ácido fólico $(\mu \mathrm{g})$ & 520 & $-8,24$ & $>98 \%$ \\
\hline
\end{tabular}




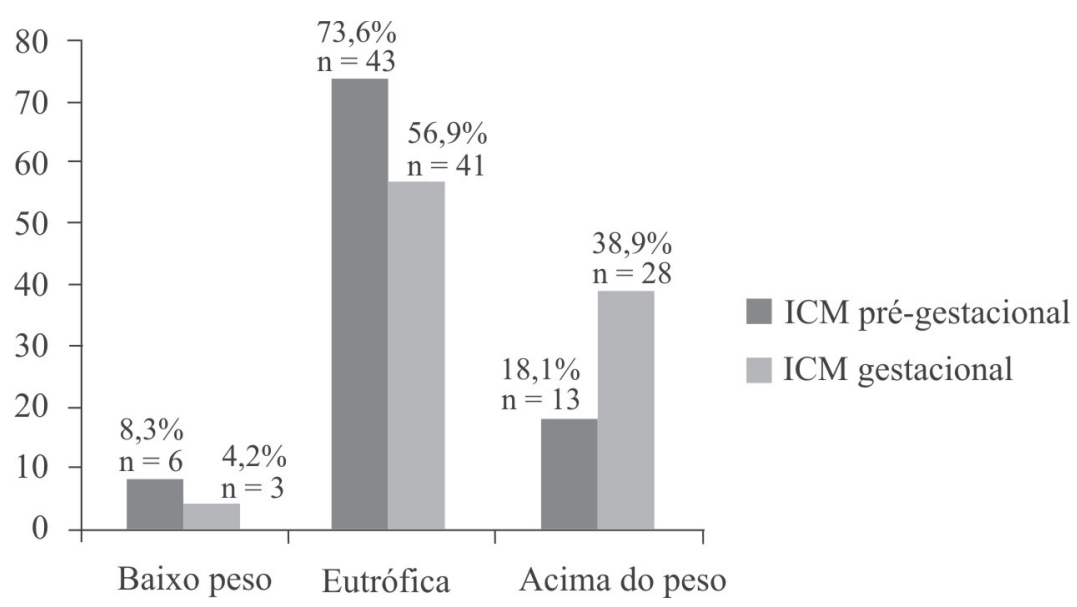

Figura 1 - Estado nutricional pré-gestacional e gestacional de gestantes adultas atendidas em Unidades Básicas de Saúde. Picos-PI, 2011.

IMC: Índice de Massa Corporal.

\section{DISCUSSÃO}

Sabe-se que o crescimento e o desenvolvimento fetal normal dependem de fatores biológicos, patológicos e socioeconômicos. Entre os fatores biológicos, destaca-se a idade materna; entre os patológicos, o estado nutricional materno inadequado; e entre os fatores sociais, enfatizamse a escolaridade materna, a renda familiar e os estilos de vida inadequados, como tabagismo e consumo de bebida alcoólica $^{(6,14,15)}$.

Os resultados desta pesquisa mostram que as gestantes estão inseridas principalmente na faixa etária entre 20 e 34 anos, período considerado excelente do ponto de vista reprodutivo, uma vez que a literatura confirma que o aumento da idade materna acentua o risco de morbimortalidade perinatal $^{(14,15)}$. Quanto às variáveis sociais, verificou-se maior prevalência de gestantes com ensino médio completo e união estável. Esses são fatores de proteção para a ocorrência de recém-nascidos de baixo peso ao nascer ${ }^{(16)}$.

Observou-se um elevado percentual de mulheres com renda per capita inferior a um salário mínimo. A literatura mostra que as gestantes com baixo poder aquisitivo possuem menos acesso aos alimentos e, consequentemente, podem consumir alimentos mais calóricos, por serem mais baratos, sendo esse consumo um fator negativo para a saúde materna e para o desenvolvimento saudável do feto ${ }^{(2,15)}$. Ressalta-se que a maioria das gestantes pertence a um grupo de baixa renda per capita, o que pode explicar, em parte, a alta prevalência de gestantes com excesso de peso ${ }^{(15)}$.

Com relação ao estilo de vida, um percentual importante de gestantes admitiu fazer uso de cigarro e álcool durante a gestação. Vale destacar que o uso de substâncias nocivas à saúde e o estilo de vida pouco saudável no período gravídicopuerperal, como uso de drogas lícitas e ilícitas, devem ser investigados e desestimulados, pois crescimento fetal restrito, aborto, parto prematuro, deficiências cognitivas no concepto, mortalidade infantil, entre outros, podem estar associados a esses comportamentos inadequados ${ }^{(2,17)}$.

No presente estudo, a quantidade de gestantes que relatou consumir bebida alcoólica durante a gestação foi relevante, entretanto, destaca-se que não foi avaliada a frequência desse consumo, que poderia ser eventual. Esse consumo eventual poderia reduzir a prevalência da ingestão de álcool na população avaliada $^{(18)}$, entretanto, os dados do presente estudo estão de acordo com outras pesquisas que mostram variação do uso de álcool na gestação - de $2,0 \%$ a 20,4\% . O consumo de álcool na gestação pode ser associado ao maior risco de o bebê nascer com baixo peso $(\mathrm{OR}=4,20)^{(19,20)}$.

O consumo médio calórico não ajustado foi de 1600,3 $\mathrm{kcal} /$ dia. O baixo consumo calórico e a discrepância com a alta prevalência de excesso de peso podem ser explicados pelo sub-relato da ingestão alimentar. Estudo realizado com o método da água duplamente marcada, considerado padrão-ouro para detecção do gasto energético, mostra uma importante discrepância nos resultados dos inquéritos dietéticos, os quais, de maneira geral, subestimam a ingestão energética, em especial pelo sub-relato dos indivíduos ${ }^{(21)}$. Parte desse erro deve-se também às tabelas de composição de alimentos, que são limitadas.

Com relação à prevalência de inadequação do consumo de vitamina $\mathrm{C}$ no presente estudo, observou-se uma proximidade com os resultados de outros trabalhos ${ }^{(22,23)}$, os quais verificaram inadequação de consumo de vitamina 
$\mathrm{C}$ em apenas $0,8 \%$ das gestantes. Em contrapartida, a inadequação de consumo de vitamina $\mathrm{E}$ foi bem maior, semelhante ao encontrado em estudo com gestantes espanholas, cuja inadequação foi de $67,8 \% \%^{(23)}$. A baixa inadequação de vitamina $\mathrm{C}$ pode ser explicada por estarmos em um país tropical, onde há uma grande variedade de frutas cítricas, que são as principais fontes de vitamina $\mathrm{C}$, sendo confirmada por análise qualitativa das dietas, que mostrou o consumo frequente de algumas frutas ricas nessa vitamina e comuns na região, como acerola, caju, laranja, goiaba e limão.

A elevada inadequação no consumo de ácido fólico entre as gestantes é preocupante, pois baixas concentrações de ácido fólico materno podem acarretar em anemia megaloblástica, parto prematuro, baixo peso ao nascer e defeitos do tubo neural ${ }^{(24)}$. Um estudo com gestantes espanholas no primeiro trimestre da gestação mostrou que a prevalência de inadequação de consumo de ácido fólico foi de $99,6 \%$, porém, quando a avaliação de suplementos na dieta foi avaliada, uma importante redução na inadequação de consumo foi observada, reduzindo-a para $40,8 \%{ }^{(23)}$.

É importante lembrar que a elevada inadequação de consumo de ácido fólico pode estar superestimada, visto que no Brasil há uma legislação específica para a fortificação de farinhas e, consequentemente, produtos de panificação com ferro e ácido fólico, o que poderia não estar contemplado na composição da tabela estrangeira utilizada no software Nutwin, utilizado na quantificação dos nutrientes consumidos.

Com relação ao consumo de ferro dietético, observouse que menos de $2 \%$ das gestantes apresentou consumo adequado. A baixa ingestão de ferro está associada diretamente à anemia por deficiência de ferro. Nos países em desenvolvimento, a deficiência de micronutrientes na gestação é comum e aproximadamente $50 \%$ das mulheres grávidas são anêmicas. Mulheres severamente anêmicas têm risco aumentado de morte na gravidez. Treze por cento das mortes maternas na Ásia e 4\% na África são diretamente causadas pela anemia, pois esta contribui com mais de $30 \%$ das mortes por hemorragias ${ }^{(25)}$.

No Brasil, os estudos sobre prevalência de anemia em gestantes são restritos a populações específicas, não permitindo generalização dos achados ${ }^{(26)}$. No entanto, vale considerar que, no presente estudo, estimou-se a prevalência de inadequação de ferro a partir de dados dietéticos somente de fonte alimentar, não sendo considerada a suplementação medicamentosa.

A inadequação no consumo de cálcio entre as gestantes foi elevada. Convém informar que não foi considerado o cálcio suplementado, entretanto, observou-se que o consumo médio de cálcio foi de $416 \mathrm{mg} / \mathrm{dia}$, e esse baixo consumo pode se associado à baixa renda das gestantes. Os grupos populacionais de baixa renda não têm acesso aos alimentos que são fonte de cálcio, contribuindo para que não atinjam uma ingestão $\operatorname{adequada}^{(27)}$. Na gestante, a deficiência desse nutriente está associada à hipertensão gestacional, doença que, se não tratada, pode levar ao parto prematuro, comprometendo o bem-estar da mãe e do bebê. Além disso, pode haver aumento da mobilização do estoque de cálcio ósseo materno e da ocorrência de osteoporose pósmenopausa $^{(28)}$.

A elevada inadequação de consumo alimentar de vitamina A nas gestantes avaliadas mostra a grande deficiência nas escolhas alimentares desse grupo. Estudo que investigou o consumo alimentar de gestantes das áreas urbana e rural da China verificou que a adequação de consumo de vitamina A foi de $94,3 \%$ para as gestantes de área urbana, e de $65,2 \%$ para as gestantes da área rural $^{(29)}$. O resultado divergente é decorrente de diferenças metodológicas na avaliação da adequação de consumo alimentar, pois em nosso estudo foi utilizada a EAR, enquanto no outro foi usado AI.

O diagnóstico dietético, apesar das limitações inerentes aos métodos e instrumentos disponíveis, assume posição de destaque como indicador de risco nutricional e, quando utilizado em conjunto com outros indicadores de maior sensibilidade, pode contribuir para o diagnóstico e combate de carências nutricionais específicas ${ }^{(30-32)}$. A busca ou aprimoramento de métodos de inquéritos dietéticos capazes de classificar populações e indivíduos segundo categorias de risco de deficiência, para um ou mais nutrientes, certamente vem contribuindo para melhorar a relação entre o indicador dietético e os demais indicadores do estado nutricional ${ }^{(30-32)}$.

Uma limitação deste trabalho foi a impossibilidade de avaliar a adequação de proteínas e lipídeos, porque não foi possível estabelecer os valores mínimo e máximo da distribuição desses macronutrientes a partir dos valores de Estimated Energy Requirement (EER) e Acceptable Macronutrient Distribution Range (AMDR). Para calcular a EER para cada trimestre seria preciso a aplicação de 3 recordatórios nos 3 momentos da gestação $\left(1^{\circ}, 2^{\circ}\right.$ e $3^{\circ}$ trimestres). No caso dos micronutrientes, não houve esse viés porque os valores de EAR não variam de acordo com o trimestre da gestação.

A presente investigação revelou que as gestantes de Picos-PI apresentaram prevalência de inadequação da ingestão de cálcio, ferro, ácido fólico e vitamina E maior que $98 \%$, além da ingestão de energia acima das necessidades em $38,9 \%$. Esses resultados podem ser influenciados pela baixa renda per capita e mostram a necessidade da criação de estratégias que trabalhem a importância da alimentação adequada e, consequentemente, melhorem o desfecho da gestação. 
O grupo pesquisado apresentou prevalência elevada do consumo excessivo de energia, segundo o estado nutricional na fase gestacional. Esses dados são relevantes e demonstram a importância do controle e acompanhamento nutricional das gestantes, pois a epidemia da obesidade é um problema de importância mundial, com um profundo impacto sobre a saúde materno-fetal. $\mathrm{O}$ excesso de peso nos períodos prégestacional e gestacional provoca complicações na gravidez (como diabetes gestacional, hipertensão e pré-eclâmpsia), afeta o crescimento fetal, provoca anomalias congênitas e aumenta o risco da mortalidade perinatal. Hoje, sabese que o impacto da obesidade materna se estende para além da vida intrauterina e neonatal, pois atinge infância, adolescência e idade adulta ${ }^{(33)}$.

Portanto, é fundamental o desenvolvimento de estratégias de orientação nutricional com vistas a melhorar o aporte desses micronutrientes, desde a concepção e o reforço de ações de normatização de propagandas e produtos voltados a esses grupos.

\section{CONCLUSÃO}

Houve elevada prevalência de inadequação no consumo de micronutrientes (cálcio, ferro, ácido fólico e vitamina E) nas gestantes, bem como uma elevada inadequação no consumo energético, apontada pela elevada prevalência de excesso de peso nas gestantes atendidas em Unidades Básicas de Saúde. Uma situação preocupante, visto que esses resultados podem desencadear consequências adversas para o binômio mãe-filho.

\section{AGRADECIMENTOS}

Ao Hildegardes Gomes de Medeiros Borges, da Secretaria Municipal de Saúde de Picos, por permitir o recrutamento das voluntárias nas Unidades Básicas de Saúde de Picos-PI.

\section{REFERÊNCIAS}

1. Wu G, Imhoff-Kunsch B, Girard AW. Biological Mechanisms for Nutritional Regulation of Maternal Health and Fetal Development. Paediatr Perinat Epidemiol. 2012;26 Suppl 1:4-26.

2. Santiago SE, Park GH, Huffman KJ. Consumption habits of pregnant women and implications for developmental biology: a survey of predominantly Hispanic women in California. Nutr J. 2013;12(1):114.

3. Imdad A, Bhutta ZA. Nutritional management of the low birth weight/preterm infant in community settings: a perspective from the developing world. J Pediatr. 2013;162(3 Suppl):S107-14.

4. Watson PE, McDonald BW. The association of maternal diet and dietary supplement intake in pregnant New Zealand women with infant birthweight. Eur J Clin Nutr. 2010; 64(2):184-93.

5. Christian P, Stewart CP. Maternal micronutrient deficiency, fetal development, and the risk of chronic disease. J Nutr. 2010;140(3):437-45.

6. Ovesen P, Rasmussen S, Kesmodel U. Effect of prepregnancy maternal overweight and obesity on pregnancy outcome. Obstet Gynecol. 2011;118(2 Pt 1):305-12.

7. Universidade Federal de São Paulo. Programa de Apoio à Nutrição Nutwin versão 1,5. São Paulo: Departamento de Informática em Saúde, Escola Paulista de Medicina; 2002.

8. Universidade Estadual de Campinas - UNICAMP, Núcleo de Estudos e Pesquisas em Alimentação NEPA. Tabela brasileira de composição de alimentos - TACO [Internet]. $2^{\mathrm{a}}$ ed. Campinas: UNICAMP; 2006 [acesso em 2011 Abr 12]. Disponível em: http://www. unicamp.br/nepa/taco/

9. Institute of Medicine, Food and Nutrition Board. Dietary Reference Intakes. The Essential Guide to Nutrient Requirements. Washington: National Academy Press; 2006.

10. Institute of Medicine. Dietary Reference Intakes for Calcium and Vitamin D. Washington: National Academy Press, 2011. Disponível em:www.nap.edu.

11. Slater B, Marchioni DL, Fisberg RM. Estimando a prevalência da ingestão inadequada de nutrientes. Rev Saúde Pública. 2004;38(4):599-605.

12. Institute of Medicine, Subcommittee on Nutritional Status and Weight Gain During Pregnancy. Nutrition during pregnancy: part I, weight gain; part II, nutrient supplements. Washington: National Academy Press; 1990.

13. Brasil. Resolução CNS n ${ }^{\circ} 466$, de 12 de Dezembro de 2012. Aprova diretrizes e normas regulamentadoras de pesquisa envolvendo seres humanos. Diário Oficial da União, Brasília, p. 59, 2013 Jun 13, n. 12, Seção 1.

14. de Weger FJ, Hukkelhoven CWPM, Serroyen J, te Velde ER, Smits LJ. Advanced maternal age, short interpregnancy interval, and perinatal outcome. Am J Obstet Gynecol. 2011;204(5):421.e1-9.

15. Mahanta LB, Roy TD, Dutta RG, Devi A. Nutritional 
status and the impact of socioeconomic factors on pregnant women in Kamrup district of Assam. Ecol Food Nutr. 2012;51(6):463-80.

16. Santos NLAC, Costa MCO, Amaral MTR, Vieira GC, Bacelar EB, Almeida AHV. Gravidez na adolescência: análise de fatores de risco para baixo peso, prematuridade e cesariana. Ciênc Saúde Coletiva. 2014;19(3):719-26.

17. Puig C, Vall O, García-Algar O, Papaseit E, Pichini S, Saltó E, Villalbí JR. Assessment of prenatal exposure to tobacco smoke by cotinine in cord blood for the evaluation of smoking control policies in Spain. BMC Pregnancy Childbirth. 2012;12(26):1-8.

18. Silva I, Quevedo LA, Silva RA, Oliveira SS, Pinheiro RT. Associação entre abuso de álcool durante a gestação e o peso ao nascer. Rev Saúde Pública. 2011;45(5):8649.

19. Aliyu MH, Wilson RE, Zoorob R, Brown K, Alioc AP, Clayton $\mathrm{H}$, et al. Prenatal alcohol consumption and fetal growth restriction: Potentiation effect by concomitant smoking. Nicotine Tob Res. 2009;11(1):36-43.

20. Adusi-Poku Y, Edusei AK, Bonney A, Tagbor H, Nakua E, Otupiri E. Pregnant women and alcohol use in the Bosomtwe district of the Ashanti region-Ghana. Afr J Reprod Health. 2012;16(1):55-60.

21. Raymond NC, Peterson RE, Bartholome LT, Raatz $\mathrm{S}$, Jensen MD, Levine JA. Comparisons of Energy Intake and Energy Expenditure in Obese Women with and Without Binge Eating Disorder. Obesity. 2012;20(4):765-72.

22. Sukchan P, Liabsuetrakul T, Chongsuvivatwong V, Songwathana P, Sornsrivichai V, Kuning M. Inadequacy of nutrients intake among pregnant women in the deep south of Thailand. BMC Public Health, 2010;10(572): $1-8$.

23. Rodríguez-Bernal CL, Ramón R, Quiles J, Murcia M, Navarrete-Muñoz EM, Vioque J, et. al. Dietary intake in pregnant women in a Spanish Mediterranean area: as good as it is supposed to be? Public Health Nutr. 2013;16(8):1379-89.

24. Van Uitert EM, Steegers-Theunissen RP. Influence of maternal folate status on human fetal growth parameters. Mol Nutr Food Res. 2013;57(4):582-95.

25. United Nations Children's Fund - UNICEF. The State of the World's Children. New York: UNICEF; 2008.

26. Araújo CRMA, Uchimura TT, Fugimori E, Nishida FS, Veloso GBL, Szarfarc SC. Níveis de hemoglobina e prevalência de anemia em gestantes atendidas em unidades básicas de saúde, antes e após a fortificação das farinhas com ferro. Rev Bras Epidemiol. 2013;16(2):535-45.

27. ImdadA, BhuttaZA. Effects of calcium supplementation during pregnancy on maternal, fetal and birth outcomes. Paediatr Perinat Epidemiol. 2012;26 Suppl 1:138-52.

28. Olausson H, Goldberg GR, Laskey MA, Schoenmakers I, Jarjou LMA, Prentice A. Calcium economy in human pregnancy and lactation. Nutr Res Rev. 2012;25(1):4067

29. Gao H, Stiller CK, Scherbaum V, Biesalski HK, Wang Q, Hormann E, et al. Dietary intake and food habits of pregnant women residing in urban and rural areas of Deyang City, Sichuan Province, China. Nutrients, 2013; 5(8):2933-54.

30. Slater B, Enes CC, Lopez RVM, Damasceno NRT, Voci SM. Validation of a food frequency questionnaire to assess the consumption of carotenoids, fruits and vegetables using the method of triads. Cad Saúde Pública 2010; 26(11):2090-100.

31. Voci SM, Slater B, Silva MV, Marchioni DML, Latorre MRDO. Estudo de Calibração do Questionário de Frequência Alimentar para Adolescentes - QFAA. Ciênc Saúde Coletiva. 2011;16(4):2335-43.

32. Voci SM, Enes CC, Romero A, Slater B. Estimativa de valores corrigidos e o efeito da correção pelo erro de medida em dados dietéticos obtidos por Questionário de Frequência Alimentar para Adolescentes (QFAA). Ciênc Saúde Coletiva. 2012;17(2):463-71.

33. Tenenbaum-Gavish K, Hod M. Impact of Maternal Obesity on Fetal Health. Fetal Diagn Ther. 2013;34(1):1-7.

\section{Endereço do primeiro autor:}

Kaoma Suzamar Silva Lacerda

Universidade Federal do Piauí

Rua Cícero Eduardo, s/n

Bairro: Junco

CEP: 64600-000 - Picos - PI - Brasil

E-mail: kaomalacerda@hotmail.com

Endereço de correspondência:

Joilane Alves Pereira Freire

Universidade Federal do Piauí

Departamento de Nutrição

Rua Cícero Eduardo, s/n

Bairro: Junco

CEP: 64600-000 - Picos - PI - Brasil

E-mail: joilane@hotmail.com 